\title{
Changing the policy for intermittent preventive treatment with sulfadoxine- pyrimethamine during pregnancy in Malawi
}

\author{
Chikondi A. Mwendera ${ }^{1}$, Christiaan de Jager ${ }^{{ }^{*}}$, Herbert Longwe ${ }^{2}$, Kamija Phiri ${ }^{3}$, Charles Hongoro ${ }^{1,4}$ \\ and Clifford M. Mutero ${ }^{1,5}$
}

\begin{abstract}
Background: The growing resistance of Plasmodium falciparum to sulfadoxine-pyrimethamine (SP) treatment for uncomplicated malaria led to a recommendation by the World Health Organization for the use of artemisinin-based combination therapy. Inevitably, concerns were also raised surrounding the use of SP for intermittent prevention treatment of malaria during pregnancy (IPTp) amidst the lack of alternative drugs. Malawi was the first country to adopt intermittent prevention treatment with SP in 1993, and updated in 2013. This case study examines the policy updating process and the contribution of research and key stakeholders to this process. The findings support the development of a malaria research-to-policy framework in Malawi.
\end{abstract}

Methods: Documents and evidence published from 1993 to 2012 were systematically reviewed in addition to key informant interviews.

Results: The online search identified 170 potential publications, of which eight from Malawi met the inclusion criteria. Two published studies from Malawi were instrumental in the WHO policy recommendation which in turn led to the updating of national policies. The updated policy indicates that more than two SP doses, as informed by research, overcome the challenges of the first policy of two SP doses only because of ineffectiveness by $P$. falciparum resistance and the global lack of replacement drugs to SP for IPTP.

Conclusion: International WHO recommendations facilitated a smooth policy change driven by motivated local leadership with technical and financial support from development partners. Policy development and implementation should include key stakeholders and use local malaria research in a research-to-policy framework.

Keywords: Malaria, Pregnancy, Sulfadoxine-pyrimethamine, Policy change, Malawi

\section{Background}

The resistance of the malaria parasite to anti-malarial drugs has led to expensive policy changes in many countries causing strain on available resources [1]. Although the World Health Organization (WHO) is instrumental in guiding health policy development, contextual factors unique to different countries need to be assessed before adopting and implementing these recommendations [2].

\footnotetext{
*Correspondence: tiaan.dejager@up.ac.za

${ }^{1}$ School of Health Systems and Public Health, Institute for Sustainable Malaria Control (UP ISMC), University of Pretoria, Private Bag X363, Pretoria 0001, South Africa

Full list of author information is available at the end of the article
}

Policy development is a tedious process that requires an understanding of the institutional and individual actors and of the context in which the process occurs [3]. Walt and Gilson [4] described this relationship when they developed a health policy framework that explores the context, content, and processes in which actors are engaged. Andersen [5] developed a framework for understanding the policy process incorporating problem identification, agenda setting, policy formulation, policy adoption, policy implementation and policy evaluation.

Malawi established its National Malaria Control Programme (NMCP) in 1984 [6]. The NMCP based their first five-year implementation plan (1985-1989) on WHO 
recommendations for malaria control in areas with proven chloroquine $(\mathrm{CQ})$ resistance in Africa [7]. One of its policies was to provide CQ chemoprophylaxis to special groups of individuals including pregnant women. In response to the growing evidence of CQ resistance, studies assessed alternative drugs to replace CQ. Malawi invested tremendously in research on malaria in pregnancy and became the first country to adopt intermittent preventive treatment in pregnancy with sulfadoxinepyrimethamine (IPTp-SP) in 1993 [8, 9]. The policy recommended that pregnant women should receive two doses during pregnancy with the first dose being given at the first antenatal visit after the first trimester of pregnancy (typically 16th to 22nd week of gestation) and the second dose at the beginning of the third trimester (between 28 and 34 weeks of gestation) [10]. Over time, growing $P$. falciparum resistance to SP for the treatment of uncomplicated malaria led to the WHO recommendation of switching to artemisinin-based combination therapy (ACT) [11] and related concerns were raised about the efficacy of SP for IPTp. The WHO convened an Evidence Review Group (ERG) on IPTp-SP in 2012 which reviewed various research evidence relevant to updating the IPTp policy. The ERG acknowledged that more than two doses of IPTp-SP would be more beneficial than the usual two doses that were previously administered [8]. The ERG recommended that IPTp-SP be given at each antenatal visit, with the first dose given early in the second trimester and subsequent doses given at monthly intervals up to the time of delivery. Following these recommendations, in 2013 the NMCP in Malawi adapted its IPTp-SP policy by recommending that women should receive at least three doses of SP during pregnancy [12].

The process of updating the IPTp-SP policy was examined with the aim of understanding policy development. The role of stakeholders and relevant research evidence during the policy development in Malawi was also assessed.

\section{Conceptual framework}

This case study forms part of a larger effort to understand policy development and the role of relevant research in this process in order to develop a framework that can facilitate the use of evidence from malaria research for policy formulation in Malawi. One important aspect of policy analysis is to understand the involvement of stakeholders and research in the process while considering the various factors that govern the need for the policy [13]. This study was conceptualized on the premise that different factors besides overwhelming evidence may influence policy development. The Walt and Gilson policy analysis framework [4] stipulates that aside from content analysis, the actors, processes and the context in which policy change occurs are required for policy analysis. The policy analysis was supplemented by the Andersen's model of policy cycle [5].

\section{Methods}

Mixed methods in form of a systematic review of published evidence, a review of key documents and key informant in-depth interviews (KIIs) were utilized in the policy analysis to enable triangulation.

\section{Systematic review}

This method aimed to establish the availability of local evidence likely to be used in the policy change process. Relevant articles were sought by searching the references of all reviewed articles. Combinations of the following specific key words relating to IPTp-SP were searched by using the medical subject heading (MESH) strategy: sulfadoxine-pyrimethamine (SP), Fanasil, pyrimethamine drug combination, pregnancy, and Malawi. Articles published between 1993 and 2012 were searched to capture all studies conducted in Malawi related to IPTp-SP from inception to the time of the policy update. These studies were assumed to provide timely evidence and were more likely to be included in the policy development process. The following combinations were used during the search: ("pregnancy" [MeSH Terms] OR "pregnancy" [All Fields]) AND ("fanasil, pyrimethamine drug combination" [Supplementary Concept] OR "fanasil, pyrimethamine drug combination" [All Fields] OR "sulfadoxine-pyrimethamine" [All Fields]) AND ("Malawi" [MeSH Terms] OR "Malawi" [All Fields]) AND (“1993/01/01" [PDAT]: “2012/12/31” [PDAT]). We searched the MEDLINE (Ovid), PubMed, Scopus and Cochrane Library databases.

\section{Selection criteria}

From the articles identified by the systematic review above, studies selected for analysis were based on the following criteria: (1) conducted in Malawi between 1993 and 2012; (2) evaluating two doses of IPTp-SP; (3) evaluating three or more IPTp-SP doses; (4) assessing two versus three or more IPTp-SP doses. The selection was limited to studies assessing the optimal response of $P$. falciparum infection to IPTp-SP by excluding studies conducted on HIV-positive women. HIV infection reduces the ability of a pregnant woman to control the malaria infection resulting in a suboptimal response to IPTp-SP [14]. Two independent co-authors, CM and HL, judged the eligibility of the studies and resolved disagreements by consensus.

\section{Document review}

Available documents such as reports, circulars, directive letters and minutes from meetings conducted during 
the policy development process were sought to provide a forum for triangulation, to verify the stakeholders and to verify important dates and events throughout the process. WHO IPTp policy documents $[8,15]$ and local IPTp-SP policy documents [12] were reviewed to examine the extent to which they referenced research evidence during policy development.

\section{Key informant interviews}

KIIs with key stakeholders involved in policy development comprising malaria researchers/advisors, policy makers, and programme/project coordinators were conducted. Interviewees participated in the policy updating process, and their views were considered to capture what transpired and general experiences on the change. Purposive sampling identified key informants. Fifteen individuals were identified and interviewed. Six interviewees were senior malaria-in-pregnancy researchers and advisors, three interviewees were policy makers and six interviewees were programme/project coordinators. Table 1 summarizes the experience, expertise and role played by each key informant (KI) during the policy changes.

The principal investigator conducted all the interviews, probing and exploring in-depth issues based on the conceptual framework of the study. The interviews were conducted in English using a semi-structured interview tool.

\section{Themes covered in the in-depth interviews}

The participants were asked to narrate their memories of the policy process by contemplating the question: "Can you please describe the process by which the IPTp-SP policy change occurred in Malawi?”
Interviews covered specific themes that included: (1) context in which the policy occurred; (2) opportunities during the policy process; (3) challenges encountered during the policy process and (4) lessons learned.

\section{Data management and analysis}

The recordings were transcribed and coded based on the themes, and the software Nvivo 11 was used to organize the data, while verbatim quotes illustrated concepts, supported conclusions and brought reality to the situation. The Giorgi's phenomenological approach, which focuses on the experiences of participants with shared life experiences, was used. This approach documents the findings from the interviewee's point of view, collecting their descriptions of their lived world on the interpretation of the meaning of the described phenomena [16].

\section{Results}

Systematic review

One hundred and seventy potential publications were identified using database searches of which eight publications from Malawi were selected using the inclusion criteria and subsequently reviewed (Fig. 1).

\section{Description of the publications}

Eight studies that met the inclusion criteria were reviewed (Table 2). Verhoeff et al. [17] measured the parasite prevalence in mothers who received one, two, or three SP doses during pregnancy and the associated incidence of low birth weight (LBW) in infants. Although there was no significant difference in peripheral or placental blood parasite prevalence, the mean birthweights

Table 1 Details of key informants (KIs) involved in the policy update for intermittent preventative treatment during pregnancy with sulfadoxine-pyrimethamine (IPTp-SP) for malaria in Malawi

\begin{tabular}{|c|c|c|c|c|}
\hline KI & Sex & Expertise & Experience & Role \\
\hline 1 & Male & Malaria epidemiologist & Over 15 years in malaria research & Researcher/advisor \\
\hline 2 & Male & Medical epidemiologist & Over 10 years in malaria research & Researcher/advisor \\
\hline 3 & Female & Malaria epidemiologist & Over 30 years in malaria research & Researcher/advisor \\
\hline 4 & Male & Clinician and malaria epidemiologist & Over 40 years in malaria research & Researcher/advisor \\
\hline 5 & Male & Malaria epidemiologist & Over 10 years malaria research & Researcher/advisor \\
\hline 6 & Male & Senior malaria scientist & Over 40 years in malaria research & Researcher/advisor \\
\hline 7 & Female & Clinical epidemiologist & 6 years & Policy maker \\
\hline 8 & Male & Malaria in pregnancy coordinator & Over 5 years in malaria research & Policy maker \\
\hline 9 & Male & Chief of health services & Five years & Policy maker \\
\hline 10 & Female & Malaria in pregnancy coordinator & Over 5 years in malaria research & Programme/project coordinator \\
\hline 11 & Male & Malaria advisor & 15 years & Programme/project coordinator \\
\hline 12 & Male & Malaria advisor & 5 years & Programme/project coordinator \\
\hline 13 & Male & Policy development and analysist & 4 years & Programme/project coordinator \\
\hline 14 & Male & Malaria advisor & 5 years & Programme/project coordinator \\
\hline 15 & Male & Malaria program specialist & 20 years & Programme/project coordinator \\
\hline
\end{tabular}




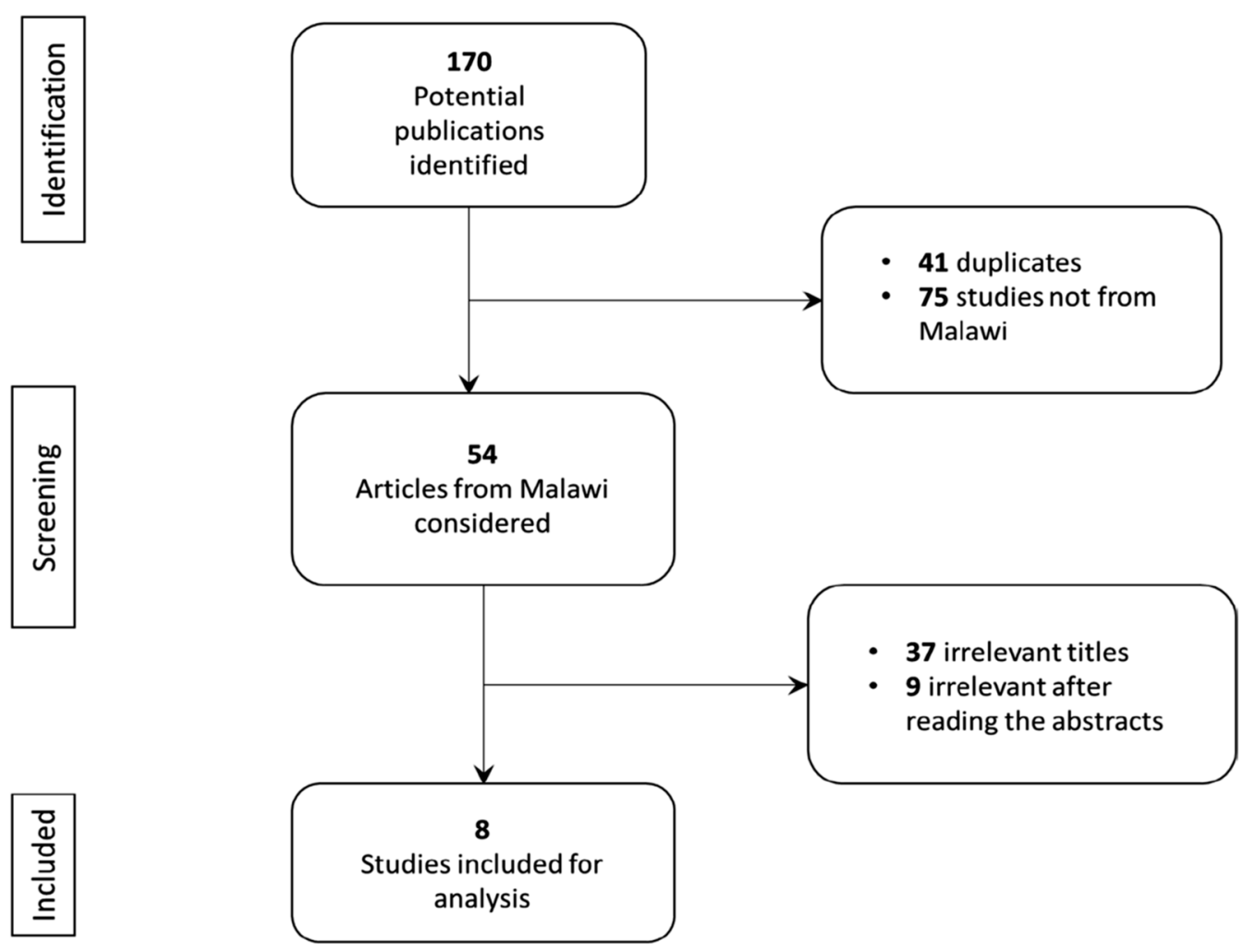

Fig. 1 A flow chart of the selection process for studies reviewed

of infants were higher, resulting in a decrease of LBW babies born to mothers that received two or more SP doses. Taylor et al. [18] explored the link between IPTpSP, the presence of resistant parasite at delivery and multiple measures of adverse delivery outcome. Receiving SP as IPTp did not raise pregnancy-associated malaria despite increasing prevalence and fixation of SP-resistant $P$. falciparum. Although $\mathrm{LBW}$ prevalence was lower $(11.8 \%)$ in the full IPTp group than in the suboptimal group $(\mathrm{P}=0.48)$, the difference was not significant. Taylor et al. [18] recommended the modified regimen of IPTp-SP for comprehensive antenatal care. Rogerson et al. [19] assessed the relationship between the number of IPTp-SP doses and various health indicators. Placental malaria prevalence decreased from $31.9 \%$, in women who did not receive SP, to $22.8 \%$ in women with $\geq 2$ SP-doses, while LBW prevalence decreased from $23 \%$ (no IPT) to $10.3 \%$ (IPTp-SP) in the two groups of women. Rogerson et al. [19] recommended that IPTp-SP should be continued based on the positive impact, but that researchers continuously evaluate treatment. Filler et al. [14] assessed the efficacy of monthly SP compared to the two doses of SP in preventing placental malaria in both HIV positive and negative women. HIV negative women who received a monthly dose of SP had a lower (2.3\%) incidence of placental malaria compared to women who received two doses (6.3\%). Filler et al. [14] recommended that areas of intense falciparum transmission should adopt a monthly IPTp-SP regimen. This study was included in the metaanalysis that led to the WHO policy recommendation $[15,20]$.

Several similar studies have assessed the efficacy of the monthly SP dose. Luntamo et al. [21] compared the effect of monthly SP, or monthly SP and two doses of azithromycin (AZI-SP) to the standard 2-SP regimen in preventing preterm deliveries and LBW. Preterm incidence was $17.9 \%$ in controls (2-SP), $15.4 \%$ in the monthly group $(\mathrm{P}=0.32)$ and $11.8 \%$ in the AZI-SP group $(P=0.01)$. There was a lower risk of LBW in the AZI-SP group $(0.61, P=0.02)$ and the monthly SP group $(0.71, \mathrm{P}=0.71)$ compared to the control group. Luntamo et al. [21] concluded that AZI-SP reduces the incidence of preterm delivery and LBW under certain conditions. Luntamo et al. [22] compared the effect of monthly SP, or monthly SP and two doses of azithromycin (AZI-SP) and a standard 2-SP dose (control) on malaria at delivery. HIV-negative women that received a monthly dose of SP $(0.26, \mathrm{P}<0.0001)$ and those that received the AZI-SP regimen $(0.249, \mathrm{P}<0.0001)$ had a significantly lower risk of malaria compared to the control group. Luntamo et al. 


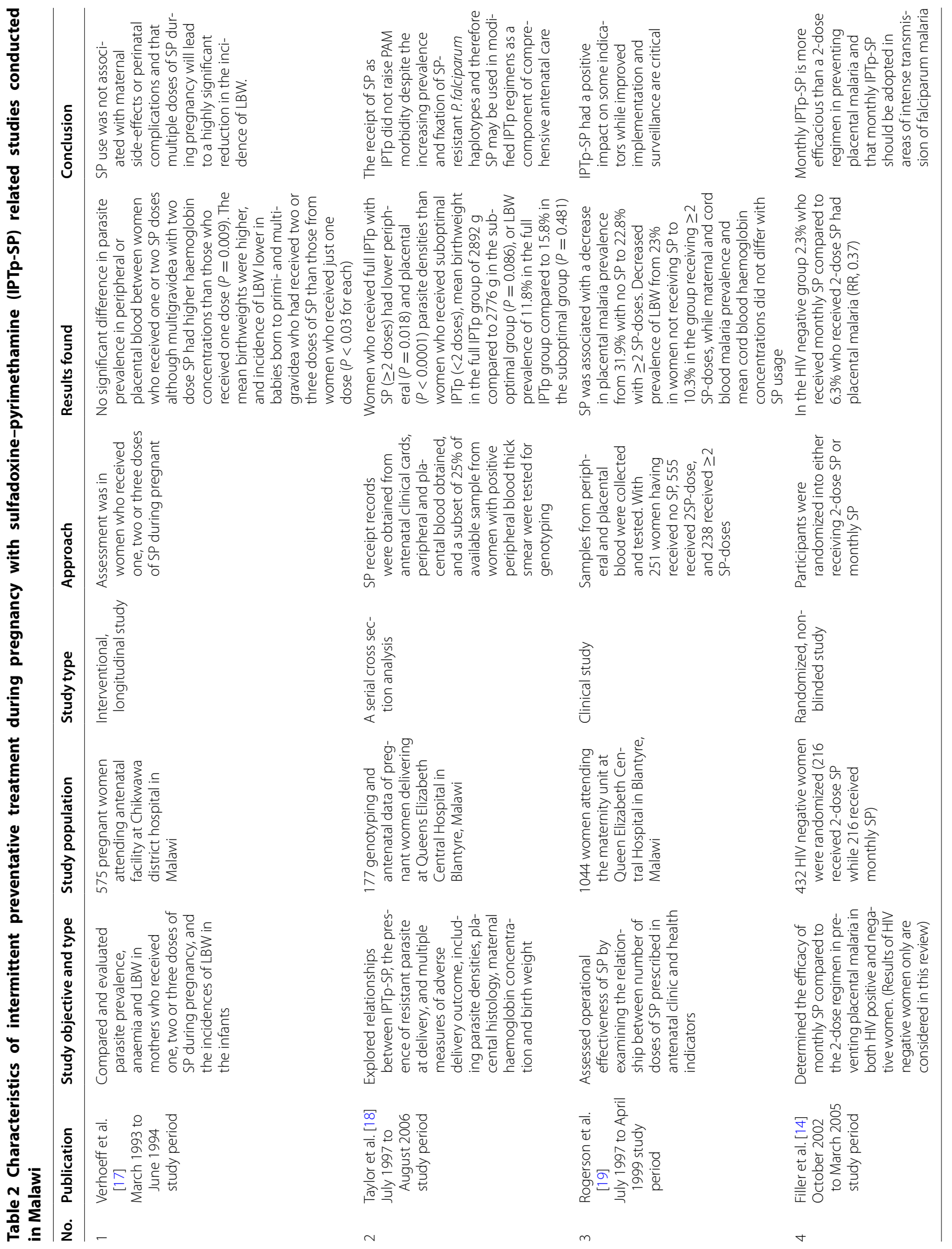




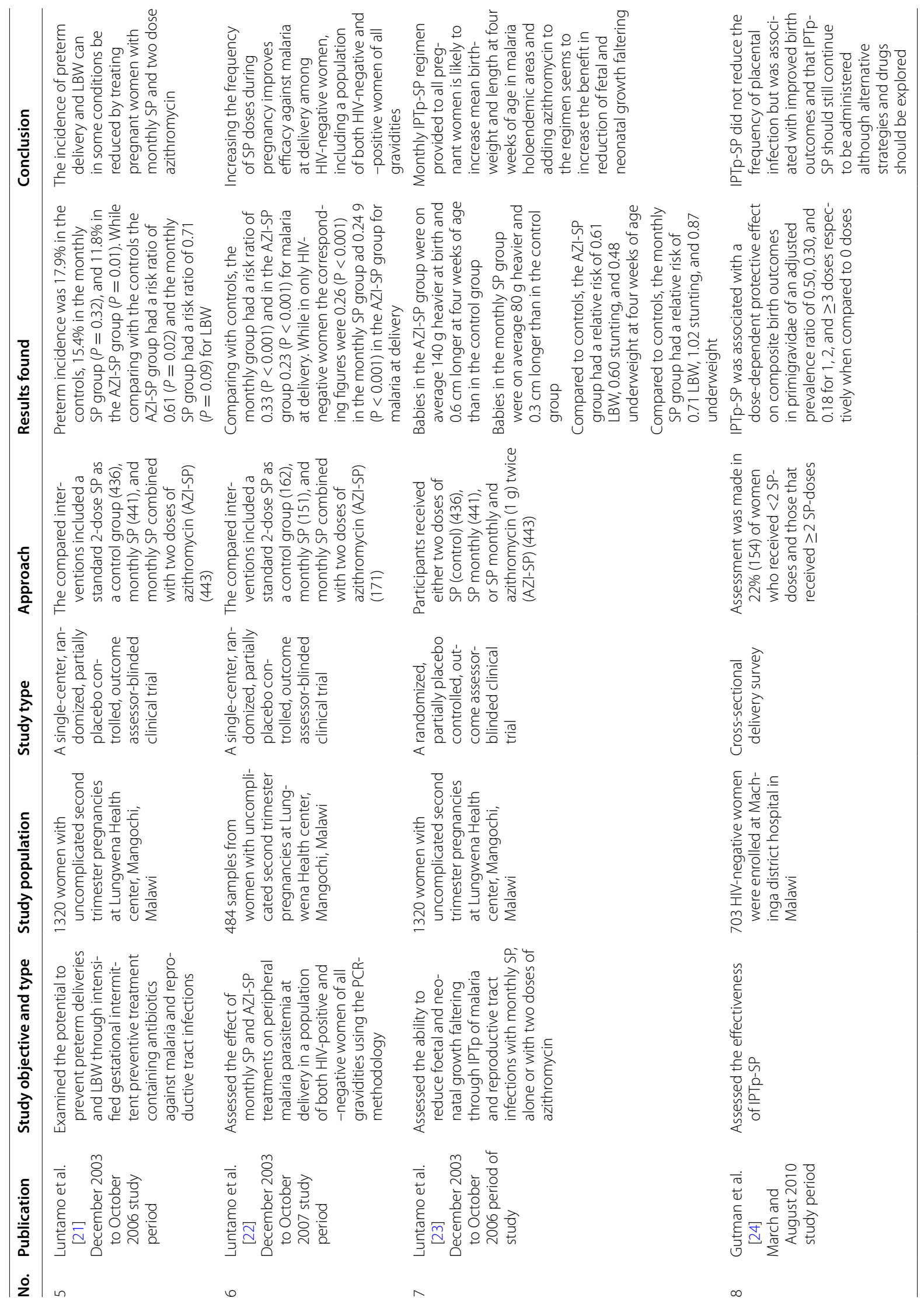


[22] recommended that frequency of SP doses during pregnancy should be increased; these recommendations were included in the meta-analysis leading to amended WHO policy recommendations $[15,20]$. Luntamo et al. [23] compared the effect of monthly SP, or AZI-SP and a standard 2-SP dose (control) on foetal and neonatal growth. Pregnant women who received monthly SP had babies with heavier mean birthweights that were taller at four weeks of age, and the addition of azithromycin further increased the benefits in reducing growth faltering. Gutman et al. [24]. assessed the effectiveness of IPTp-SP on placental infection and composite birth outcomes. Their findings showed that IPTp-SP was associated with a dose-dependent protective effect on composite birth outcomes but did not reduce the frequency of placental infection. They recommended that IPTp-SP be given while exploring alternative strategies and drugs.

\section{Document review}

Policy documents were examined to assess how research evidence was used and included in WHO documents [8, $15]$ and the local treatment policy [12]. The WHO documents used a variety of evidence from across the globe upon which they based their policy formulation. Evidence from Malawi was instrumental in agenda setting $[9,25]$ and policy development $[14,18,19,21-23,26]$. The local treatment policy document did not have a formal reference section which hampered the assessment of used evidence. However, the authors of the policy document acknowledged the importance of the WHO recommendations during its development.

\section{Stakeholders and their roles during the IPTp-SP policy change}

The Ministry of Health $(\mathrm{MOH})$ (represented by the NMCP and the Reproductive Health Directorate (RHD)), the National Malaria Advisory Committee (NMAC); Malaria Care, Clinton Health Access Initiative (CHAI), WHO; Support for Service Delivery Integration-Services (SSDI-services), malaria researchers, and PMI/USAID were primary stakeholders in the policy change process. Stakeholders gave technical advice, developed guidelines, reviewed and edited guidelines, trained health workers, implemented policies in health facilities, and provided financial support for conferences and other resources (Table 3).

\section{The process of change}

The policy change began in July 2012 when the WHO ERG made a recommendation to the Malaria Policy Advisory Committee (MPAC) for an interim policy on IPTp-SP [8]. The recommendation was adopted after an assessment of a systematic review and meta-analysis
[20]. The ERG based their recommendations on findings that associated three or more SP doses for IPTp with increased mean birth weight and reduced risk of LBW births. Based on the reviewed evidence, the ERG recommended IPTp-SP for all pregnant women with the first dose administered at antenatal visits as early as possible in the second trimester, and the subsequent doses spaced no less than four weeks apart up to the time of delivery [8]. Following this meeting in October 2012, WHO updated its IPTp-SP policy, and in April 2013 a policy brief was issued to support dissemination and urge national health authorities to adopt and implement the new recommendations.

\section{Steps for IPTp-SP policy change in Malawi}

Based on emerging literature and WHO recommendations, the NMCP updated malaria treatment guidelines that incorporated rectal and injectable artesunate, malaria rapid diagnostic tests (RDTs) for quick diagnosis of uncomplicated malaria, and the new IPTp-SP policy. Malawian policy makers took the opportunity to adapt the IPTp-SP policy given the challenges experienced during the implementation of the previous policy. The implementation of the previous policy was hampered by health workers' confusion surrounding the delivery of the second dose of SP [27]. The updated WHO recommendations do not specify the number of doses, but highlight that SP should be provided to pregnant women at each scheduled antenatal visit after the first trimester up until the time of delivery. The adapted Malawi IPTp-SP policy indicates that pregnant women should receive at least three doses of SP after the first trimester and with the last dose given close to the time of delivery. The new recommendation was strategically planned to coincide with the WHO initiative of integrating IPTp-SP into focused antenatal care (FANC) services that recommend at least four scheduled antenatal care visits [15].

In May 2013, the NMAC convened to vet the treatment guidelines before the Minister of Health and the Secretary for Health approved the new guidelines. Initially, Malaria in Pregnancy (MIP) formed part of the treatment guidelines in Malawi, and it was deemed vital to isolate and develop specific guidelines for MIP. In June 2013, a group comprising of the NMCP, RHD, and PMI/USAID, coordinated by the SSDI-services, convened for one week to develop MIP guidelines and revise the MIP training manual for health workers following the WHO recommendations. The guidelines were approved in July 2013 [12].

From October to November 2013, two Trainer of Trainers (TOTs) workshops were conducted in the northern and central eastern zones of the country to orient TOTs on the updated malaria case management 
Table 3 Summary of activities provided by key stakeholders involved in the policy updating process for intermittent preventative treatment during pregnancy with sulfadoxine-pyrimethamine (IPTp-SP) for malaria in Malawi

\begin{tabular}{|c|c|c|}
\hline Stakeholder & Main responsibility & Role in policy change \\
\hline NMCP & $\begin{array}{l}\text { Development of malaria policies, and implementation of } \\
\text { malaria programs }\end{array}$ & Drafting of guidelines, leading the process, and finalization of guidelines \\
\hline $\mathrm{RHD}$ & $\begin{array}{l}\text { Implementation of reproductive health services in the } \\
\mathrm{MOH}\end{array}$ & Drafting of the guidelines, and policy implementation \\
\hline SSDI-services & $\begin{array}{l}\text { Effective integration and delivery of quality services under } \\
\text { the Malawi Essential Health Package (EHP), and to } \\
\text { strengthen the national health system in line with the } \\
\text { National Health Sector Strategic Plan for 2011-2016 }\end{array}$ & $\begin{array}{l}\text { Coordination of activities, drafting of guidelines, finalizing, printing, dis- } \\
\text { semination of guidelines, and training of health workers }\end{array}$ \\
\hline $\mathrm{WHO}$ & Provision of technical advice and recommendation & $\begin{array}{l}\text { Overseeing of the whole process in accordance to WHO recommenda- } \\
\text { tions }\end{array}$ \\
\hline PMI/USAID & Provision of technical and financial support for the NMCP & Provided financial support for all activities and provided technical advice \\
\hline NMAC & $\begin{array}{l}\text { Provide expert opinion to the NMCP in policy and pro- } \\
\text { gramme development }\end{array}$ & Vetting and final approval of the guidelines \\
\hline Malaria Care & Provision of malaria diagnostic and treatment services & Training of health workers \\
\hline $\mathrm{CHAl}$ & Strengthening of integrated health systems & Revision of case management guidelines, training of health workers \\
\hline $\begin{array}{l}\text { Malaria } \\
\text { researchers }\end{array}$ & $\begin{array}{l}\text { Conducting malaria research to provide evidence and } \\
\text { guide policy formulation }\end{array}$ & Provided technical review of evidence and guidelines \\
\hline
\end{tabular}

NMCP National Malaria Control Programme, RHD Reproductive Health Directorate, SSDI support for service delivery integration, $P M I$ President's Malaria Initiative, USAID United States Agency for International Development, NMAC National Malaria Advisory Committee, CHAI Clinton Health Access Initiative, MOH Ministry of Health, WHO World Health Organization

guidelines and new IPTp-SP policy. The TOTs were immediately required to roll out training sessions for other health workers in their work places. Despite this requirement, training sessions for health workers only commenced in August 2014. The delay in transition was due to a change in the per diem policy by the government through the Office of President and Cabinet (OPC) on all Developing Partner (DP) programmes in Malawi. The per diem policy changed regarding direct payments to service providers for costs such as accommodation. Participants did not receive sitting allowances and were not paid if they conducted duties for which they received a salary during the training session. These adjustments meant that participants were not able to pay for necessities if they travelled to different training venues. Logistical issues were resolved by conducting training sessions in areas where most of the participants were situated, and the new policy was implemented in August 2014. Evaluation of the new IPTp-SP policy is yet to be conducted. Figure 2 illustrates the timeline of policy change process, and the roles played by stakeholders based on Andersen's model of policy change [5].

\section{Context of policy change}

In 2007, Malawi changed its treatment policy for uncomplicated malaria from SP to artemether-lumefantrine. The change of drug regimen was complicated as it attracted many activities which required approval by the Ministry of Health. The new treatment guidelines for
IPTp-SP were introduced in 2013 and was uncomplicated by comparison. Updating the policy did not draw any official launch since it was approved along with the treatment policy. This offered an enabling environment for a smooth transition of the IPTp-SP policy change as narrated below:

"In fact there is no need for launching when it's a revised policy but if it's a new policy that's when the launching comes in" (Programme/Project Coordinator)

The policy update aimed to overcome a flaw of the old 2-IPTp-SP policy. Health workers were confused about the exact time to give the second dose mainly due to late antenatal attendance by women leading to the programme failing to meet the $80 \%$ Roll Back Malaria coverage target [27]. The new policy was adapted to address this challenge as highlighted below:

"We noted a big challenge that there was a misunderstanding in terms of application by the service providers because it [the policy] was saying at 13 weeks give the first dose of SP then the second dose at 28 weeks...so people just complied to those dates...so if one comes at 18th week or 20th week then [they] will not be given SP and who comes at 32 weeks would not be given the second dose...so the previous guidelines made some limitation and that was our main challenge for low coverage but this policy change to 3 doses or more means that a preg- 


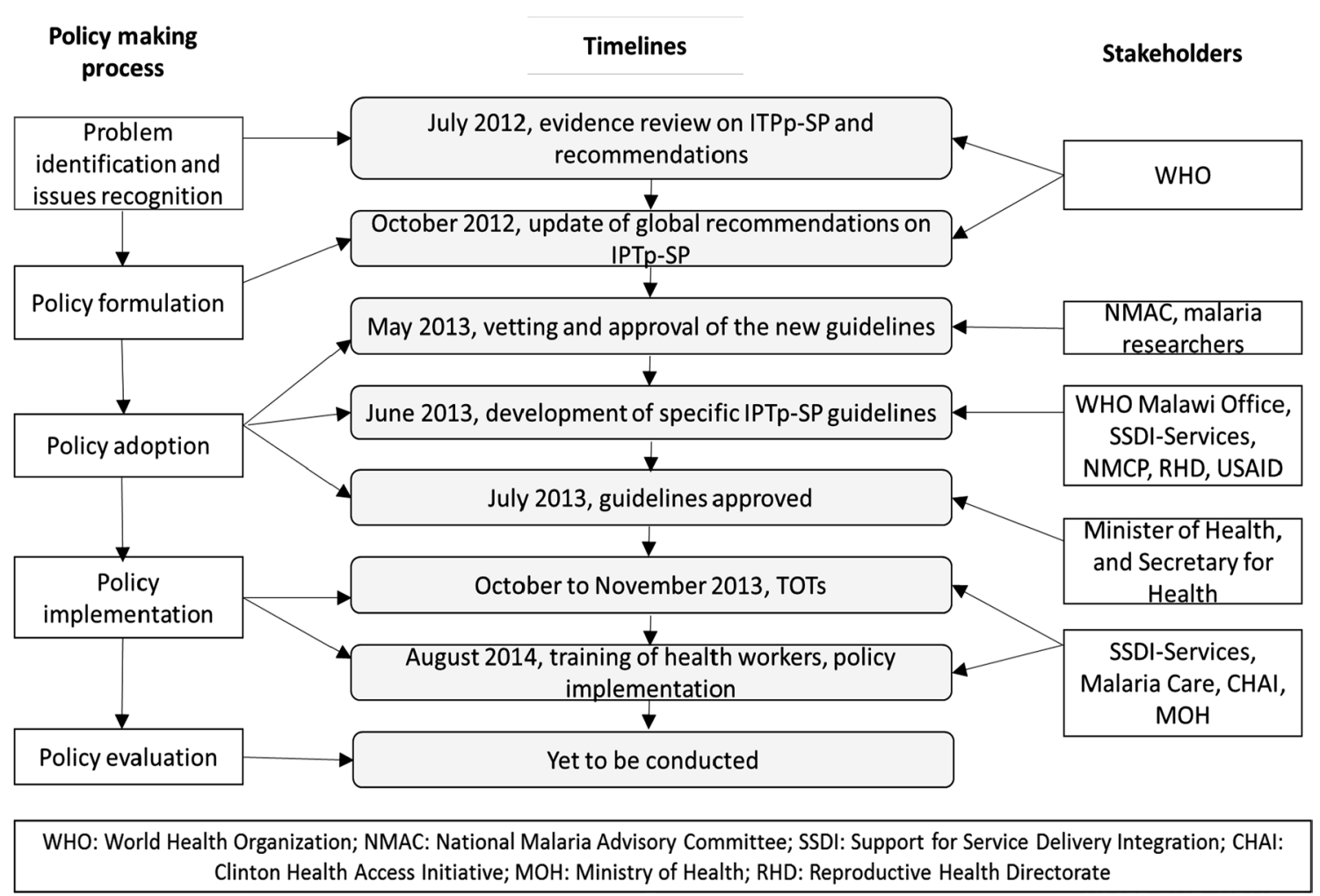

Fig. 2 The policy making process, timeline of events and stakeholders involved in the policy updating process for intermittent preventative treatment during pregnancy with sulfadoxine-pyrimethamine (IPTp-SP) for malaria in Malawi

nant woman can get IPTp even after 36 weeks...there are no restrictions so that's one of the advantages". (Programme/Project Coordinator)

The global lack of an alternative drug to replace SP for IPTp meant that increasing the doses, as stipulated by evidence, was the only tangible alternative. The challenges of coverage (as highlighted above), lack of an alternative drug and new evidence on dosage increment provided an ideal environment for IPTp-SP policy change.

"We do not have any other alternative that's why they are recommended that we should just use 3 or more doses but studies by College of Medicine are underway to explore other drugs". (Programme/Project Coordinator)

\section{Opportunities for smooth policy transition}

Participants revealed that the availability of technical and funding support from partners (USAID/PMI and SSDIservices) supported the completion of policy updating process. This was acknowledged as follows:
"Funding component and technical support was assured by the project...but also PMI who are the funders of the project... a malaria section...I think they have been very supportive....I think they wanted this to succeed as such their pressure made it easier for us to move forward". (Programme/Project Coordinator)

The relatively low cost of SP facilitated smooth policy transition. The low cost of SP meant that increasing doses would not incur a heavy cost, and that partners were willing to fund the policy implementation.

"SP is frankly pretty [an] inexpensive drug...so we can usually cover the entire need for a few hundred thousand dollars a year...compared to ACTs and RDTs and others". (Programme/Project Coordinator)

The WHO policy recommendations, based on a robust evidence review, also facilitated smooth policy transition. Such a consensus makes it easier for countries to adopt as stipulated below:

"WHO has also been pushing that we change... and the policy brief was backed by a lot of scientific evidence that was done extensively across the globe... so 
that push from these global stakeholders also enabled us to work fast". (Programme/Project Coordinator)

"Actually we just adapted the WHO guidelines... revised the malaria treatment guidelines accordingly...that was really straight forward". (Researcher/ Advisor)

The NMCP, who are the key stakeholders in implementing malaria control interventions in the country, had a vested interest in updating the policy. The NMCP made all efforts from within the government to adopt and implement the policy change. This was confirmed below:

"Overall the National Malaria Control Programme who are the mandated programme to look at malaria, also had keen interest for this to happen and be implemented". (Programme/Project Coordinator)

The inclusion of relevant stakeholders at the beginning of the process of change was key in driving the policy update. The inclusion of RHD, who implement IPTp as an integrated reproductive health service, was strategic at policy implementation stage, as described below:

"I need to point out that the reproductive health directorate [as a] key department in malaria in pregnancy issues were also very supportive...you know we cannot talk of malaria in pregnancy without the reproductive health directorate because [it] is a platform that we use to implement IPTp". (Programme/Project Coordinator)

\section{Challenges encountered in the policy process}

The NMCP were mandated to develop the new IPTpSP as a stand-alone malaria control policy. The previous IPTp guidelines were embedded as a component within the malaria treatment guidelines. Developing new standalone IPTp-SP guidelines was a cumbersome process that had to incorporate information that was previously part of the Malaria Treatment guidelines, as describe below:

"But now malaria in pregnancy were embedded in malaria case management..... and what was lacking in those documents were the detailed health education that goes with it...financing, partnership... all those things were missing because it was only considered as a treatment component not necessarily as a strategy...so the challenge was that we had to develop [the] guidelines from a scratch". (Programme/Project Coordinator)
Developing separate guidelines meant that health workers needed to be reoriented to consider IPTp-SP as a preventive strategy and an integrated case management tool. As acknowledged by a stakeholder that it was also difficult to bring together various stakeholders to one gathering and commit their time to developing the guidelines:

\section{"To get different stakeholders come together and agree on something it takes time because people have got a lot of demands on their work...so for them also to dedicate their time to this, it's a little bit of time". (Programme/Project Coordinator)}

Orientation of health workers occurred in stages, one district at a time. Thus other districts were still implementing the old policy while training was taking place. This was partly due to funding partners' policies to release funds in stages. Concerns were raised as below:

"This time around we are conducting cascade training not as the way we always do, because things change, partners change the way of doing business, so as we are going down to the districts to do the actual trainings it will be a little bit slow because others are still using the two dose........because of the way we are implementing due to funders money. But otherwise in a nutshell we just believe that by the end of the year we [will] have finished and the whole country is [will] implement one policy." (Policy maker)

In addition to this challenge was the delay in training health workers due to the change in per diem policy by the government on all Developing Partner (DP) programmes in Malawi.

\section{Lessons learnt during the policy change}

Participants highlighted that dedication to the policy process is critical, especially government commitment. Partners can provide resources but if the $\mathrm{MOH}$ as owners of health policies are not motivated, the process will face challenges. A stakeholder confirmed this:

"The most important element for a policy to be effectively developed, the relevant government department should have the interest in that policy and they drive the whole process.... the NMCP team were so keen to have this done...that's why we didn't find a lot of problems". (Programme/Project Coordinator)

Another important factor highlighted by participants was the availability of local evidence on which to base changes. An advisor revealed that research evidence forms the foundation for further policy changes: 
"Normally when we want to effect a policy change there must have been a study that was conducted or an assessment that was conducted...so that has always been the trend in Malawi that we are guided by studies". (Researcher/Advisor)

Participants stressed that resources should be available at all stages from policy adoption to implementation to achieve smooth policy change, as highlighted below:

\section{"When you are embarking on policy change you should have everything available, you should have the money for the change, for everything that means the drugs themselves, for the guideline change, infor- mation to the general public because you can have the money to do the trainings but if you don't inform the public it is very difficult for them to welcome the intervention quickly. So when you have all this together the policy change is very smooth". (Policy maker)}

Participants acknowledged the significance of involving relevant stakeholders in the policy process ranging from funding partners, policy makers in the $\mathrm{MOH}$, Policy implementers, and the public.

\section{Discussion}

The reduced efficacy of SP in the treatment of uncomplicated malaria led to its replacement with $\mathrm{ACT}$ as recommended by WHO [28]. Inevitably, concerns were raised about the continued use of SP for IPTp. Following these concerns, IPTp-SP was extensively monitored to evaluate its use or explore alternative drugs. Recently a study conducted in Malawi proposing intermittent screening and treatment with dihydroartemisin-piperaquine (ISTp-DP) as an alternative to IPTp-SP did not show superiority in both parasite clearance and birth outcomes. Thus it recommended continued use of SP for IPTp [29]. Whilst alternative drugs or strategies for IPTp have not yet been found, evaluations of IPTp-SP have revealed that giving SP to all pregnant women at each antenatal care visit from early in the second trimester, with subsequent doses spaced four weeks apart up to the time of delivery, is beneficial for birth outcomes [8]. Concerns of increased doses of SP for IPTp were raised on the uptake of folic acid and iron during pregnancy because of SP is a folate antagonist. However, evidence has shown that there is no interference with SP when the right doses of 30-60 mg of element iron plus $0.4 \mathrm{mg} /$ day folic acid supplementation are administered $[8,30]$.

Comprehensive studies conducted in Malawi have contributed to the body of knowledge on IPTp and have informed local and international IPTp policies. Upon implementation of the first global IPTp-SP policy [6],
Malawi immediately started monitoring the policy and confirmed the safety of SP in IPTp and established that multiple doses of SP during pregnancy led to a highly significant reduction in the incidence of LBW [17]. The two SP dose regimen remained unchanged despite these results. Increased $P$. falciparum resistance to SP in the treatment of uncomplicated malaria led to similar concerns regarding the use of SP in IPTp. Several studies monitored the effects of IPTp-SP during pregnancy and birth outcomes. These studies revealed the positive outcomes of IPTp-SP and recommended that more SP doses would have further positive results [14, 18, 19, 21-24]. Despite this evidence originating in Malawi, IPTp-SP policy changes were only made after the amended WHO recommendation was released in 2013. The two studies conducted in Malawi $[14,21]$ were included in the metaanalysis that led to the change in WHO recommendations [20] which then informed local policy changes. Nevertheless, the WHO does not impose recommendations since countries are at liberty to adopt or adapt them as has been the case for Malawi.

The policy review process in Malawi was largely based on WHO recommendations $[8,15]$ which incorporated evidence from Malawi for agenda setting $[9,25]$ and policy development $[14,18,19,21-23,26]$. The lack of references in the local policy document [12] hampered the assessment of the degree to which local research was consulted [31]. While the availability of evidence is one content factor that needs to be considered when making a policy [3], the policy process often overlooks actors, processes and contextual factors [4]. In Malawi, the involvement of the right stakeholders during the policy process was strategic. Although the NMCP is the overall coordinating body for malaria interventions, the inclusion of the RHD was vital since they are responsible for delivery of reproductive health services in the country including IPTp-SP. The importance of this collaboration can never be over-emphasised by the malaria in pregnancy working group meeting in Kenya, which attracted both NMCP and reproductive health $\mathrm{MOH}$ country representatives to discuss the Roll Back Malaria guidelines for MIP [32]. Funding partners in the process played a major role through tracking progress and obtaining firsthand reports.

Changing the policy was met with technical and administrative challenges that included the tedious process of developing new IPTp-SP guidelines, bringing together all stakeholders in one place, and the cascade training of health workers which was adversely affected by change of per diem policy by the government. The previous per diem policy left room for public funds abuse when among other things events such as training workshop were conducted away requiring participants to 
claim for some expenses not incurred and it was widely viewed as a method of supplementing one's salary. The change in policy hence led to boycott of such events until resolutions were sought and one of the logistical issues was resolved by conducting training workshops in areas where most participants resided.

Nonetheless, many opportunities facilitated a smooth policy process. Most important was leadership by the $\mathrm{MOH}$ through the NMCP that showed keen interest in seeing the policy developed and implemented. Similarly, the NMCP was identified as critical in driving government efforts in engaging and collaborating with the right stakeholders such as researchers in seeking evidence for policy formulation and its implementation [33]. It is important that the motivation emanates from the government if such a process is to be realized. Policy change in Timor-Leste that contained an escalation of malaria cases during a crisis was similarly supported and driven by government [34]. The use of WHO recommendations as motivation for policy change facilitated support from PMI, who provided technical and funding support to the process.

\section{Conclusion}

Malawi changed its IPTp policy based on the WHO recommendation in 2012. Research conducted in Malawi was instrumental in changing the global IPTp-SP policy due to the inclusion of findings in the systematic review that led to the WHO policy change. Malawi adapted and changed its IPTp-SP policy based on the resulting WHO recommendations. This change did not face many hurdles but was a welcome opportunity to address some of the challenges faced by health workers during implementation of the previous policy. The policy updating process has highlighted the importance of commitment by the concerned government department to be motivated and drive the process. This should be accompanied by a thorough stakeholder analysis to identify and involve relevant key stakeholders from the initial stages of the policy change process. In addition, it is critical to utilize local evidence for this process and address current local health burdens leading to efficient public health care. The local evidence used in the process should be documented in the policy documents and guidelines for purposes of tracking research utilization and its impact.

Ideally, policymakers should use a framework that facilitates the use of malaria research to champion knowledge translation and work towards addressing the malaria burden in Malawi. Therefore, lessons from this study will inform the development of the malaria research-topolicy framework in Malawi and the process of developing this framework can also advise the development of research-to-policy frameworks in other settings.

\section{Abbreviations}

ACT: Artemisin-based Combination Therapy; CHAl: Clinton Health Access Initiative; CQ: Chloroquine; ERG: Evidence Review Group; WHO: World Health Organization; IPTp-SP: Intermittent Preventive Treatment in pregnancy with sulfadoxine-pyrimethamine; Klls: Key Informant Interviews; LBW: Low Birth Weight; MIP: Malaria in Pregnancy; MOH: Ministry of Health; MPAC: Malaria Policy Advisory Committee; NMCP: National Malaria Control Programme; PMI: Presidential Malaria Initiative; RHD: Reproductive Health Directorate; SSDIservices: Support for Service Delivery Integration-Services; TOTs: Trainer of Trainers; USAID: United States Agency for International Development.

\section{Authors' contributions}

CAM conceived, developed the idea, and prepared the manuscript. HL contributed to shaping of the idea. CMM, KP, TdJ, and CH contributed their technical expertise in directing and critiquing of the manuscript. All the authors contributed to the drafting of the manuscript. All authors read and approved the final mansucript.

\section{Author details}

${ }^{1}$ School of Health Systems and Public Health, Institute for Sustainable Malaria Control (UP ISMC), University of Pretoria, Private Bag X363, Pretoria 0001, South Africa. ${ }^{2}$ Mailman School of Public Health, ICAP at Columbia University, Pretoria, South Africa. ${ }^{3}$ School of Public Health and Family Medicine, College of Medicine, University of Malawi, Blantyre, Malawi. ${ }^{4}$ Population Health, Health Systems and Innovation, Human Sciences Research Council (HSRC), Pretoria, South Africa. ${ }^{5}$ International Centre of Insect Physiology and Ecology (ICIPE), P.O. Box 30772, Nairobi, Kenya.

\section{Acknowledgements}

We acknowledge the funding support from the University of Pretoria Institute for Sustainable Malaria Control (UP ISMC) and MRC Collaborating Centre for malaria research, Mrs Estelle Gobler, (librarian) for assistance in the literature search exercise, Mr John Munthali for reviewing and informing on the events of the policy process, and Dr. Cheryl Tosh for editing the manuscript.

\section{Competing interests}

The authors declare that they have no competing interests.

\section{Availability of data and materials}

The datasets generated and/or analysed during the current study are not publicly available due to keeping the identities of respondents confidential as they granted consent for interviews on the basis of remaining anonymous but are available from the corresponding author on reasonable request.

\section{Ethics approval}

Ethical clearance was obtained from the National Health Sciences Research Committee (NHSRC) in Malawi and the Faculty of Health Sciences Research Ethics Committee at the University of Pretoria (Ref No. 146/2013). Participants were also requested to provide consent approval to be interviewed and recorded before the interviews.

\section{Funding}

University of Pretoria Institute for Sustainable Malaria Control (UP ISMC) and MRC Collaborating Centre for malaria research are acknowledged for the provision of funding support toward the study.

Received: 7 January 2017 Accepted: 14 February 2017

Published online: 20 February 2017

\section{References}

1. Williams HA, Vincent-Mark A, Herrera Y, Chang OJ. A retrospective analysis of the change in anti-malarial treatment policy: Peru. Malar J. 2009;8:85.

2. Williams HA, Durrheim D, Shretta R. The process of changing national malaria treatment policy: lessons from country-level studies. Health Policy Plan. 2004;19:356-70.

3. Durrheim DN, Williams HA, Barnes K, Speare R, Sharp BL. Beyond evidence: a retrospective study of factors influencing a malaria treatment 
policy change in two South African provinces. Critical Public Health. 2003;13:309-30

4. Walt G, Shiffman J, Schneider H, Murray SF, Brugha R, Gilson L.'Doing' health policy analysis: methodological and conceptual reflections and challenges. Health Policy Plan. 2008;23:308-17.

5. Anderson JE. Public policymaking. Cengage Learn. 2014.

6. Steketee R. A decade of progress in malaria policy and program development in Malawi: 1984-1993. Washington, DC: United States Agency for International Development; 1995.

7. Malawi National Malaria Control Programme. Five year implementation plan: 1985-1989. Lilongwe: Ministry of Health; 1984.

8. WHO. Intermittent preventive treatment of malaria in pregnancy (IPTp) with sulfadoxine-pyrimethamine (SP). Geneva: World Health Organization; 2012.

9. Feng G, Simpson JA, Chaluluka E, Molyneux ME, Rogerson SJ. Decreasing burden of malaria in pregnancy in Malawian women and its relationship to use of intermittent preventive therapy or bed nets. PLOS ONE. 2010:5:e12012.

10. Schultz L, Steketee RW, Chitsulo L, Wirima JJ. Antimalarials during pregnancy: a cost-effectiveness analysis. Bull World Health Organ. 1995;73:207-14.

11. World Health Organization. Antimalarial drug combination therapy: report of a WHO Technical Consultation. Geneva: World Health Organization; 2001.

12. Malawi National Malaria Control Programme. Revised guidelines for the treatment of malaria in Malawi. Lilongwe: Ministry of Health; 2013.

13. Mubyazi GM, Gonzalez-Block MA. Research influence on antimalarial drug policy change in Tanzania: case study of replacing chloroquine with sulfadoxine-pyrimethamine as the first-line drug. Malar J. 2005;4:51.

14. Filler SJ, Kazembe P, Thigpen M, Macheso A, Parise ME, Newman RD, et al. Randomized trial of 2-dose versus monthly sulfadoxine-pyrimethamine intermittent preventive treatment for malaria in HIV-positive and HIVnegative pregnant women in Malawi. J Infect Dis. 2006;194:286-93.

15. WHO. Policy brief for the implementation of intermittent preventive treatment of malaria in pregnancy using sulfadoxine-pyrimethamine (IPTp-SP). Geneva: World Health Organization; 2013.

16. Giorgi A. Sketch of a psychological phenomenological method. Phenomenol Psychol Res. 1985;1:23-85.

17. Verhoeff F, Brabin B, Chimsuku L, Kazembe P, Russell W, Broadhead R. An evaluation of the effects of intermittent sulfadoxine-pyrimethamine treatment in pregnancy on parasite clearance and risk of low birthweight in rural Malawi. Ann Trop Med Parasitol. 1998;92:141-50.

18. Taylor SM, Antonia AL, Chaluluka E, Mwapasa V, Feng G, Molyneux ME, et al. Antenatal receipt of sulfadoxine-pyrimethamine does not exacerbate pregnancy-associated malaria despite the expansion of drug-resistant Plasmodium falciparum: clinical outcomes from the QuEERPAM study. Clin Infect Dis. 2012;55:42-50.

19. Rogerson SJ, Chaluluka E, Kanjala M, Mkundika P, Mhango C, Molyneux ME. Intermittent sulfadoxine-pyrimethamine in pregnancy: effectiveness against malaria morbidity in Blantyre, Malawi, in 1997-1999. Trans R Soc Trop Med Hyg. 2000;94:549-53.

20. Kayentao K, Garner P, van Eijk AM, Naidoo I, Roper C, Mulokozi A, et al. Intermittent preventive therapy for malaria during pregnancy using 2 vs 3 or more doses of sulfadoxine-pyrimethamine and risk of low birth weight in Africa: systematic review and meta-analysis. JAMA. 2013;309:594-604.
21. Luntamo M, Kulmala T, Mbewe B, Cheung YB, Maleta K, Ashorn P. Effect of repeated treatment of pregnant women with sulfadoxine-pyrimethamine and azithromycin on preterm delivery in Malawi: a randomized controlled trial. Am J Trop Med Hyg. 2010;83:1212-20.

22. Luntamo M, Rantala A, Meshnick SR, Cheung YB, Kulmala T, Maleta K, et al. The effect of monthly sulfadoxine-pyrimethamine, alone or with azithromycin, on PCR-diagnosed malaria at delivery: a randomized controlled trial. PLOS ONE. 2012;7:e41123.

23. Luntamo M, Kulmala T, Cheung YB, Maleta K, Ashorn P. The effect of antenatal monthly sulphadoxine-pyrimethamine, alone or with azithromycin, on foetal and neonatal growth faltering in Malawi: a randomised controlled trial. Trop Med Int Health. 2013;18:386-97.

24. Gutman J, Mwandama D, Wiegand RE, Ali D, Mathanga DP, Skarbinski J. Effectiveness of intermittent preventive treatment with sulfadoxinepyrimethamine during pregnancy on maternal and birth outcomes in Machinga district, Malawi. J Infect Dis. 2013;208:907-16.

25. Taylor SM, Antonia A, Feng G, Mwapasa V, Chaluluka E, Molyneux M, et al. Adaptive evolution and fixation of drug-resistant Plasmodium falciparum genotypes in pregnancy-associated malaria: 9-year results from the QuEERPAM study. Infect Genet Evol. 2012;12:282-90.

26. Kapito-Tembo A, Meshnick SR, van Hensbroek MB, Phiri K, Fitzgerald M, Mwapasa V. Marked reduction in prevalence of malaria parasitemia and anemia in HIV-infected pregnant women taking cotrimoxazole with or without sulfadoxine-pyrimethamine intermittent preventive therapy during pregnancy in Malawi. J Infect Dis. 2011;203:464-72.

27. Ashwood-Smith H, Coombes Y, Kaimila N, Bokosi M, Lungu K. Availability and use of sulphadoxine-pyrimethamine (SP) in pregnancy in Blantyre District: a Safe Motherhood and BIMI Joint Survey. Malawi Med J. 2002;14:8-11.

28. WHO. Guidelines for the treatment of malaria. Geneva: World Health Organization; 2006

29. Madanitsa M, Kalilani L, Mwapasa V, van Eijk AM, Khairallah C, Ali D, et al. Scheduled intermittent screening with rapid diagnostic tests and treatment with dihydroartemisinin-piperaquine versus intermittent preventive therapy with sulfadoxine-pyrimethamine for malaria in pregnancy in Malawi: an open-label randomized controlled trial. PLoS Med. 2016;13:e1002124.

30. Peters PJ, Thigpen MC, Parise ME, Newman RD. Safety and toxicity of sulfadoxine/pyrimethamine. Drug Saf. 2007;30:481-501.

31. Mwendera C, de Jager C, Longwe H, Phiri K, Hongoro C, Mutero CM. Malaria research and its influence on anti-malarial drug policy in Malawi: a case study. Health Res Pol Syst. 2016;14:1.

32. Malaria RB. Malaria in pregnancy working group: 18th annual meeting, Nairobi. Kenya: World Health Organization; 2016.

33. Mwendera CA, Jager C, Longwe H, Phiri K, Hongoro C, Mutero CM. Facilitating factors and barriers to malaria research utilization for policy development in Malawi. Malar J. 2016;15:512.

34. Martins JS, Zwi AB, Martins N, Kelly PM. Malaria control in Timor-Leste during a period of political instability: what lessons can be learned? Conflict Health. 2009;3:1.

\section{Submit your next manuscript to BioMed Central and we will help you at every step:}

- We accept pre-submission inquiries

- Our selector tool helps you to find the most relevant journal

- We provide round the clock customer support

- Convenient online submission

- Thorough peer review

- Inclusion in PubMed and all major indexing services

- Maximum visibility for your research

Submit your manuscript at www.biomedcentral.com/submit
BioMed Central 